\title{
Cycle biologique de Clinostomum golvani n. sp.
}

(Trematoda: Clinostomidae) parasite larvaire de

Biomphalaria glabrata, Mollusque vecteur de

Schitosoma mansoni en Guadeloupe

\author{
par H. NASSI * et Ch. BAYSSADE-DUFOUR ** \\ * Département de Biologie animale (Dir. Pr C. Combes), Université, \\ avenue de Villeneuve, F 66025 Perpignan Cedex. \\ ** Laboratoire de Zoologie - Vers, associé au C.N.R.S. (Dir. Pr A.G. Chabaud), \\ Muséum national d'Histoire naturelle, 43, rue Cuvier, F 75231 Paris Cedex 05.
}

RESUME. Le cycle biologique d'une nouvelle espèce de Clinostomidae, Clinostomum golvani, est décrit en Guadeloupe. Le premier hôte intermédiaire est le Mollusque vecteur de Schistosoma mansoni dans cette île, Biomphalaria glabrata, qui peut être stérilisé par ce parasite. Poecilia reticulata (guppy) est le deuxième hôte intermédiaire. Les adultes ont été obtenus expérimentalement chez Butorides virescens (l'hôte définitif naturel), Nycticorax nycticorax et Ardea purpurea. L'adulte est très proche de Clinostomum complanatum mais les stades larvaires (rédies et cercaire) présentent plusieurs différences. La chétotaxie d'une cercaire de Clinostomidae est donnée pour la première fois.

Life cycle of Clinostomum golvani n. sp. (Trematoda : Clinostomidae) a larval parasite of Biomphalaria glabrata, the snail vector of Schistosoma mansoni in Guadeloupe.

SUMMARY. The life cycle of a new species of Clinostomidae, Clinostomum golvani, is described in Guadeloupe (French West Indies). The first intermediate host is the snail vector of Schistosoma mansoni in this island, Biomphalaria glabrata, which can be sterilized by this parasite. Poecilia reticulata (guppy) serves as the second intermediate host. Adult worms were obtained under experimental conditions from Butorides

Accepté le 28 février 1980. 
virescens (the definitive host in nature), Nycticorax nycticorax and Ardea purpurea. The adult worm closely resembles Clinostomum complanatum but the larval stages (rediae and cercaria) show several differences. The chetotaxic description of a cercaria of Clinostomidae is given for the first time.

\section{Introduction}

En Guadeloupe, le vecteur de Schistosoma mansoni, Biomphalaria glabrata, est fréquemment infesté par les larves d'un Trématode Clinostomidae qui peut déterminer une stérilité durable de ce Mollusque. Dans le cadre de nos recherches sur l'utilisation éventuelle de Trématodes pathogènes en lutte biologique contre le vecteur de la Schistosomose intestinale, nous avons été amenés à étudier le cycle de ce parasite. Les adultes obtenus expérimentalement appartiennent au genre Clinostomum Leidy, 1836 et sont morphologiquement très proches de l'espèce type du genre, $C$. complanatum (Rud., 1814) Braun, 1899 (=C. marginatum (Rud., 1819) Braun, 1899, d'après Baer, 1933 ; Yamaguti, 1933 ; Dollfus, 1950, Ukoli, 1966). Cependant les différences relevées au niveau des stades larvaires entre le parasite guadeloupéen et $C$. complanatum nous paraissent suffisantes pour les considérer comme deux espèces distinctes.

\section{Matériel et méthodes}

Les Mollusques infestés ont été récoltés dans différentes stations de la partie Est de la Guadeloupe (Grande Terre) et conservés au laboratoire à la température de $25^{\circ} \mathrm{C}$. Les stades larvaires (cercaire et rédies) ont été étudiés sur le vivant. Afin de travailler dans des conditions comparables à celles de Krull (1934), les principales dimensions des cercaires ont été prises après fixation à chaud dans du formol à $5 \%$. Deux méthodes d'imprégnation argentique ont été utilisées pour l'étude chétotaxique des cercaires : celle de Chatton et Lwoff (1930) et celle de Combes et al. (1976). La deuxième permet de redresser et tourner, en vue ventrale ou dorsale, dans le milieu du montage, les cercaires courbées et couchées sur un côté; elle permet leur décapitation et l'orientation adéquate de la calotte céphalique. L'infestation du deuxième hôte intermédiaire, Poecilia reticulata (guppy), a été réalisée en exposant individuellement de jeunes poissons âgés de 1 à 2 semaines à un nombre limité de cercaires (10) émises depuis quelques minutes. Ces métacercaires obtenues expérimentalement ont donné des adultes ovigères chez Nycticorax nycticorax et Ardea purpurea. L'hôte définitif naturel, Butorides virescens, a été infesté avec des métacercaires récoltées dans la nature. Les adultes ont été étudiés in toto après fixation au liquide de Bouin et coloration au carmin boracique; leur anatomie a été précisée par l'examen de coupes sériées de $6 \mu \mathrm{m}$ d'épaisseur colorées à l'Azan die Heindenhain. 


\section{Résultats}

Adulte (fig. 1).

\section{- Clinostomidae : Clinostominae}

Morphologie et anatomie conformes à celles du genre Clinostomum. Corps massif linguiforme, 4,02 à 7,46 mm de long sur 2,33 à 3,44 mm dele large. Région pré-acétabulaire séparée du reste du corps par une constriction située approximativement à l'équa. teur acétabulaire. Rapport longueur région post-acétabulaire/région pré-acétabulaire : 2,7 à 3. Ventouse orale subterminale allongée transversalement (290 à $400 \mu \mathrm{m}$ sur 220 à $300 \mu \mathrm{m}$ ) située au centre d'un champ périoral en dépression dépourvu d'épines. Acétabulum circulaire, diamètre 600 à $850 \mu \mathrm{m}$. Epines courtes sur la totalité du tégument ventral et latéral, moins denses en arrière de la région génitale et absentes sur la face dorsale. Bouche donnant accès à un organe pharyngien (1). Caecums intestinaux à parois fortement godronnées atteignant l'extrémité postérieure du corps ; l'étude histologique montre qu'ils communiquent avec la partie distale des canaux excréteurs longitudinaux à leur débouché dans la vessie (2). Pore excréteur subterminal dorsal.

Quelle que soit la taille du spécimen, les deux testicules et l'ovaire sont compris dans le quatrième cinquième de la longueur du corps. Chez les plus petits spécimens, le bord postérieur du testicule postérieur peut même déborder dans le dernier cinquième. Testicule antérieur en grande partie situé à gauche de l'axe de symétrie du corps, 550 à $650 \mu \mathrm{m}$ de large sur 210 à $360 \mu \mathrm{m}$ de haut ; testicule postérieur à bord antérieur concave, à bord postérieur plurilobé, 780 à $800 \mu \mathrm{m}$ sur 260 à $400 \mu \mathrm{m}$. Canaux déférents naissant ventralement de la région droite de chaque testicule. Le canal postérieur qui remonte ventralement par rapport à l'ovaire et le canal antérieur. transversal, se rejoignent à la base de la poche du cirre. Partie distale de la poche du cirre s'appuyant sur la partie droite du bord antérieur du testicule antérieur. Vésicule séminale très enroulée sur elle-même ; canal éjaculateur à paroi musclée et cirre orné de papilles faisant saillie dans l'atrium génital. Orifice génital s'ouvrant sur l'axe de symétrie du corps ou légèrement à droite de celui-ci. Ovaire dorsal situé entre la poche du cirre et le testicule postérieur; ovoïde ou réniforme, il mesure 280 à $480 \mu \mathrm{m}$ dans sa plus grande dimension. Oviducte naissant sur la face interne de l'ovaire. Canal de Laurer débouchant dorsalement dans la moitié supérieure de l'espace intertesticulaire. Glande de Mehlis diffuse. L'utérus décrit plusieurs boucles dans l'espace intertesticulaire, remonte contre le bord gauche du testicule antérieur et décrit une dernière boucle avant de s'aboucher dorsalement à la base du sac utérin. Sac utérin occupant en longueur une grande partie de l'espace intercaecal, s'appuyant en arrière sur le testicule antérieur et se terminant vers l'avant à 200 ou $300 \mu \mathrm{m}$ de l'acétabulum. Les dimensions du sac utérin sont souvent réduites par l'expulsion d'une partie des œufs lorsqu'on mani-

(1) Cf. Donges, 1974.

(2) Cette particularité anatomique peu courante chez les Trématodes a déjà été signalée chez $C$. complanatum par Yamaguti (1933). 


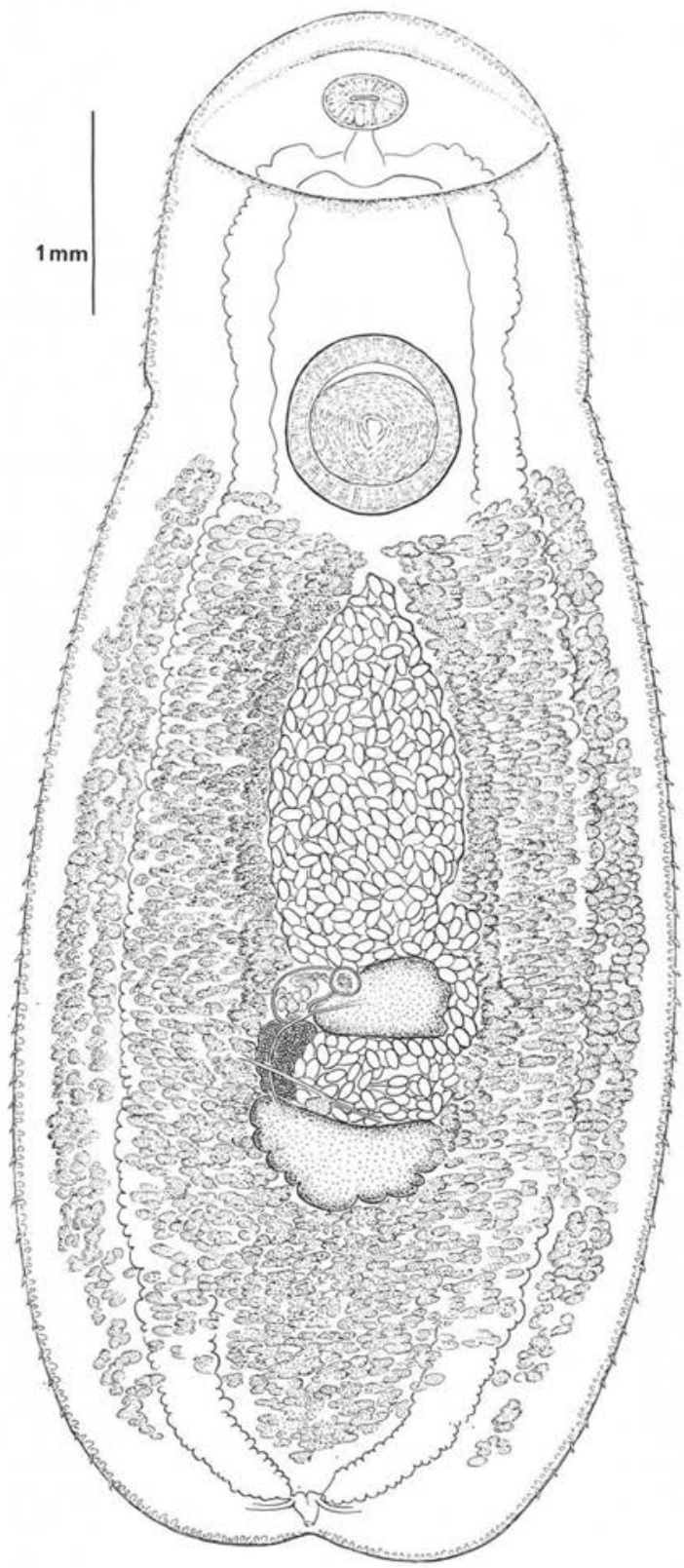

Fig. 1. Adulte de Clinostomum golvani. Face ventrale. 
pule le parasite ; cette expulsion, parfois massive, intervient peut-être dans la dissémination des œufs lorsque l'hôte définitif capture ses proies dans l'eau. Métraterme naissant ventralement à la base du sac utérin. Vitellogènes constitués de nombreux petits follicules organisés en deux champs latéraux débutant antérieurement entre l'acétabulum et le sommet du sac utérin ; ces deux champs fusionnent en arrière du testicule postérieur sans recouvrir la partie distale des caecums. Les vitelloductes transverses passent ventralement au-dessus du testicule postérieur; vitelloducte commun dilaté en un réservoir vitellin. CEufs : 111 à $120 \mu \mathrm{m}$ de long (moyenne $116 \mu \mathrm{m}$ ) sur 65 à $71 \mu \mathrm{m}$ de large (moyenne $67 \mu \mathrm{m}$ ).

\section{Hôte définitif :}

- Butorides virescens, naturel et expérimental ; Nycticorax nycticorax et Ardea purpurea (expérimental).

Localisation: Cavité buccale.

\section{Stades larvaires.}

Rédies (fig. 2, A)

De couleur jaune orangé, les rédies sont comme chez les autres Clinostomidae dépourvues d'appendices. Elles colonisent en grand nombre la région de l'ovotestis et les individus parasités sont souvent stérilisés. Dans les stades avancés de la parasitose, certaines rédies produisent à la fois des cercaires et des rédies. Chez ces jeunes rédies, à la naissance, le corps et le pharynx mesurent respectivement 250 et $32 \mu \mathrm{m}$ de long. A maturité, la longueur du corps atteint fréquemment $2000 \mu \mathrm{m}$, le maximum enregistré étant de $3100 \mu \mathrm{m}$ (pharynx : $67 \mu \mathrm{m}$ ) ; la longueur du caecum digestif est comprise entre les $2 / 3$ et les $4 / 5$ de la longueur du corps. Il y a un pore de naissance au niveau de la base du pharynx.

\section{Cercaire.}

- Morphologie générale (fig. 2, B, C)

Cercaire lophocerque ocellée. La cercaire n'a pu être observée qu'en vue latérale du fait de la compression latérale du corps et de la forte contraction de la face ventrale. Les dimensions ont été prises sur 30 corcaires fixées à chaud dans du formol à $5 \%$. Les organes non respectés par la fixation ont été mesurés sur le vivant. Corps mesurant 93 à $115 \mu \mathrm{m}$ (moyenne 100) dans sa plus granłe dimension (jusqu'à $200 \mu \mathrm{m}$ en extension maximale sur le vivant). Ocelles pigmentés cupuliformes (diamètre : $6 \mu \mathrm{m}$ ) marquant la limite du tiers antérieur du corps; leur concavité est orientée vers l'avant suivant une direction latéro-dorsale. Voile médio-dorsal débutant au niveau des ocelles et s'étendant jusqu'à l'extrémité postérieure du corps; hauteur maximale comprise entre 7 et $9 \mu \mathrm{m}$. Organe de pénétration très contractile; longueur variant de 33 à $45 \mu \mathrm{m}$. 

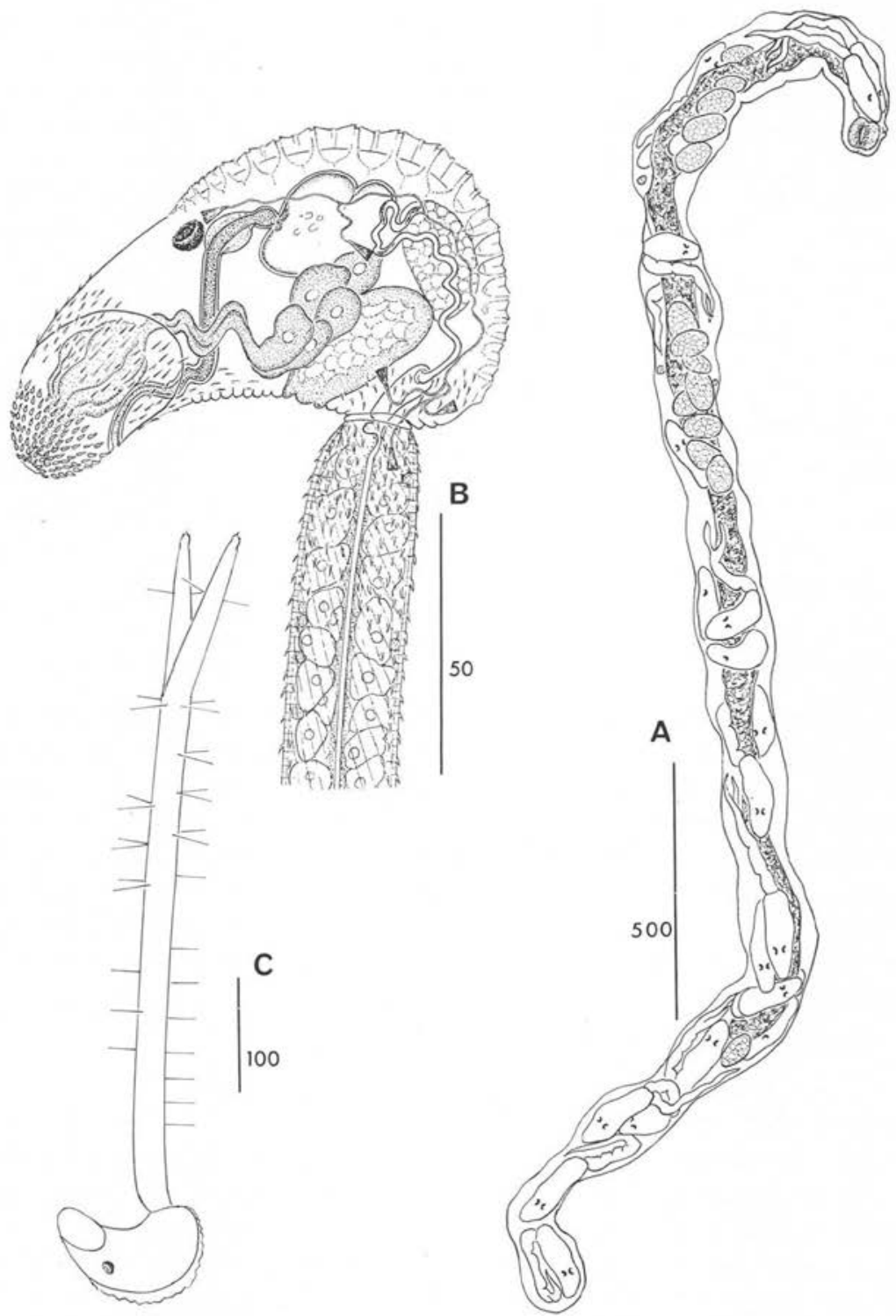

Fig. 2. A : Rédie. B : Cercaire, corps au repos et partie basale du tronc caudal en vue latérale gauche. C: Habitus de la cercaire au repos; les soies sensorielles sont représentées. Echelles en $\mu . \mathrm{m}$. 
Acétabulum non fonctionnel représenté par un massif de cellules qui, en vue latérale, est allongé en direction dorsale; grand axe mesurant de 27 à $33 \mu \mathrm{m}$. Le tégument correspondant au tiers antérieur de l'organe de pénétration présente 8 à 9 rangées d'épines coniques alternant régulièrement. Cette zone spinulée apicale est séparée par une bande de tégument nu d'une seconde zone spinulée aux épines plus fines, plus longues et à disposition moins régulière. Dorsalement cette seconde zone spinulée ne dépasse pas l'extrémité postérieure de l'organe de pénétration mais se prolonge sur la face ventrale où les épines se font plus clairsemées. La spinulation redevient relativement dense dans la région postérieure du corps. La bouche s'ouvre ventralement dans l'organe de pénétration au niveau de la bande de tégument nu. Le tube digestif d'abord ventral s'infléchit en direction dorsale; il traverse un organe ovoïde que l'on peut assimiler à un pharynx (hauteur : 12 à $13 \mu \mathrm{m}$ ) et aboutit à l'ébauche des caecums représentés par un organe sacciforme dont la face dorsale est marquée par une forte contriction transversale. Glandes de pénétration constituées par cinq paires de cellules situées entre les ébauches caecale et acétabulaire. Les canaux des cinq cellules situées de chaque côté du corps s'organisent en un faisoeau qui pénètre dans l'organe céphalique dans la région postérieure pour s'y dilater avant de déboucher à son extrémité apicale. Dorsalement, dans le tiers postérieur du corps, se trouve l'ébauche génitale constituée d'un seul massif de cellules mesurant environ $25 \mu \mathrm{m}$ dans sa plus grande dimension. Système excréteur à cinq paires de protonéphridies dont une paire localisée à la base de la queue, avec trois bouquets de cils pulsateurs dans chaque canal collecteur commun ; formule : $2[(1+1)+(1+1)+(1)]=10$. Dimensions du tronc caudal très variables sur les cercaires fixées: longueur comprise entre 270 et $370 \mu \mathrm{m}$ (moyenne : $312 \mu \mathrm{m}$ ), diamètre maximum entre 21 et $33 \mu \mathrm{m}$ (moyenne : 24,5 $\mu \mathrm{m}$ ). Fourchons : 90 à $130 \mu \mathrm{m}$ de long (moyenne : $107 \mu \mathrm{m}$ ) sur 12 à $15 \mu \mathrm{m}$ de large. La base du tronc caudal est spinulée sur une hauteur de 30 à $40 \mu \mathrm{m}$; puis jusqu'aux fourchons, la spinulation est réduite à deux rangées dorsales et deux rangées ventrales d'épines à disposition irrégulière. Les fourchons ne sont spinulés que sur les arêtes dorsales et ventrales. Le canal excréteur impair est entouré de grosses cellules de forme irrégulière ; à $30 \mu \mathrm{m}$ de l'extrémité du tronc caudal oe canal donne deux ramifications dont chacune s'ouvre à la base de la fine expansion tégumentaire qui termine chaque fourchon.

- Chétotaxie (fig. 3) :

Région céphalique (fig. $3, A, B$ ).

La région céphalique montre 16 papilles très argyrophiles disposées selon quatre cycles et ordinairement 16 éléments évaginés ou invaginés selon l'état de l'organe de pénétration; une dizaine d'entre eux, de forme ovalaire, correspondent vraisemblablement aux orifices des glandes de pénétration, les autres, environ au nombre de six, mais il peut y en avoir jusqu'à 10 , aux petites papilles situées à leur voisinage, analogues à celles décrites par J. Richard (1971) chez les cercaires de Schistosoma et de Trichobilharzia sous l'appellation CIL; la chétotaxie par hémitête est donc la suivante :

$\mathrm{CI}=1 \mathrm{CrV}, 1+3$ à $5 \mathrm{CIL}, 1 \mathrm{CID}$

$\mathrm{CII}=2 \mathrm{CII}$ 


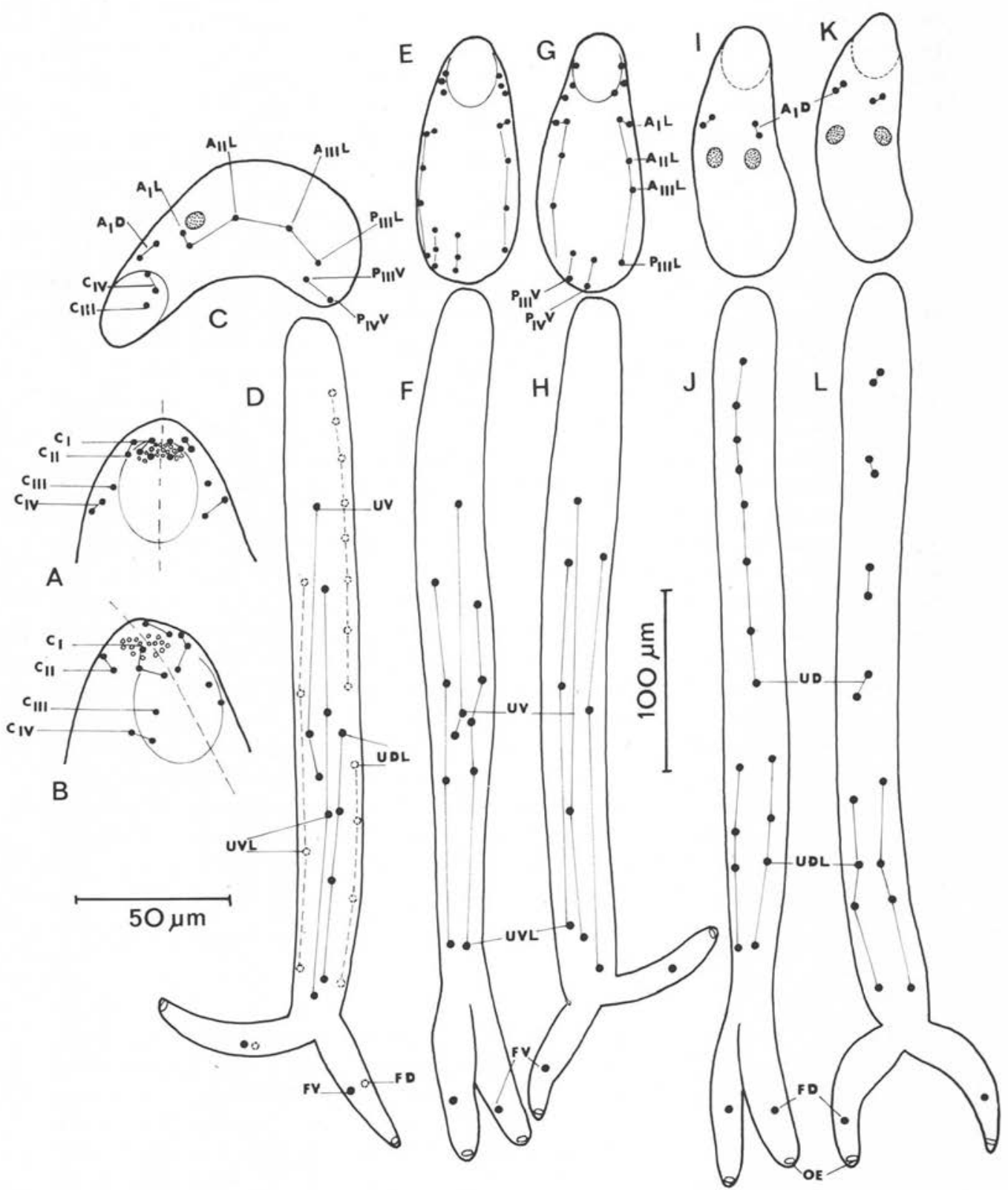

Fig. 3. Chétotaxie cercarienne. A, B : céphalique en vue ventrale. C, D : vues de profil (C, corps; D, queue). E, F, G, H : vues ventrales ( $\mathrm{E}, \mathrm{G}$, vues ventrales; $\mathrm{F}, \mathrm{H}$, queues). I, J, K, L: vues dorsales (I, K, corps; J, L, queues). 
$\mathrm{CIII}=1 \mathrm{CIII}$

$\mathrm{Crv}=2 \mathrm{CrV}$

Notons que la bouche ventrale ne s'ouvre pas au milieu des papilles Cr mais près du niveau CIII.

Région corporelle (fig. 3, C, E, G, I, K).

Les papilles ventrales sont présentes au niveau $\mathrm{P}$; les dorsales s'observent au-dessus des ocelles, en $\mathrm{AI}$; les latérales, plus nombreuses, sont distribuées régulièrement le long du corps.

$\mathrm{AI}=2 \mathrm{AIL}, 2 \mathrm{AID}$

AII $=1$ AIIL

AIII $=1$ AmL

PIII $=1$ PIIV, 1 PIIIL (peut manquer d'un côté ou peut sur certaines préparations être déportée dorsalement).

Prv $=1$ ou plus rarement 2 PrvV

Territoire du massif acétabulaire : Sans papille.

Région caudale :

- Le tronc caudal (fig. 3, D, F, H, J, L).

Le tronc caudal porte des papilles ventrales et ventro-latérales, des papilles dorsales et dorso-latérales.

$a$ - Papilles ventrales et ventro-latérales.

Deux à trois papilles médio-ventrales sont regroupées le plus souvent dans le tiers antérieur du tronc caudal, mais il arrive parfois que la dernière papille médio-ventrale se trouve au voisinage de la bifurcation caudale. Quatre ou exceptionnellement cinq paires de papilles ventro-latérales s'étagent régulièrement du niveau de la première papille médio-ventrale jusqu'à la bifurcation de la queue.

$b$ - Papilles dorsales et dorso-latérales.

Huit papilles médio-dorsales s'alignent régulièrement dans la moitié antérieure du tronc caudal; il arrive plus rarement qu'elles soient regroupées deux par deux avec un intervalle nu entre chaque groupe. Assez rarement le tronc caudal montre neuf papilles médio-dorsales. Quatre paires de papilles dorso-latérales occupent le dernier tiers du tronc caudal.

- Les furcas : chaque furca porte une papille ventrale et une papille dorsale symétriques; le pore excréteur s'imprègne à l'extrémité de chaque furca.

\section{Métacercaire}

Dans la nature les métacercaires sont hébergées par Poecilia reticulata (guppy) dont l'infestation a été réalisée expérimentalement. Les métacercaires sont localisées superficiellement dans la musculature (« yellow grub» des anglo-saxons), les Poissons 
parasités étant parfaitement visibles sur le fond sombre des mares. Le délai de maturation des métacercaires est très long. Les essais d'infestation de Nycticorax nycticorax avec des métacercaires âgées de 100 jours ont été négatifs; ces essais ont été positifs avec des métacercaires âgées de 165 à 184 jours. A ce stade de leur évolution, leur longueur est comprise entre 4 et $6 \mathrm{~mm}$. L'infestation de l'hôte définitif naturel Butorides virescens avec des métacercaires de tous âges récoltées dans la nature nous a livré des adultes chez lesquels la longueur minimum observée a été de 4,02 mm. On peut estimer que la métacercaire est infestante lorsque sa longueur est voisine de $4 \mathrm{~mm}$. Elle peut poursuivre sa croissance bien au-delà, sa longévité pouvant atteindre au moins 19 mois. La longueur maximale observée est de $8,22 \mathrm{~mm}$. Il n'y a pas de différences morphologiques ou anatomiques notables entre notre matériel et les métacercaires de $C$. marginatum dont Cort (1913) a donné une description très précise. Les métacercaires remontent dans la bouche de l'hôte définitif dans les vingt-quatre heures qui suivent l'infestation. Les Trématodes sont ovigères deux jours après leur fixation dans la bouche.

\section{Discussion}

Suivant les critères retenus dans la mise au point d'Ukoli (1966) pour la détermination des Clinostomum adultes, le parasite guadeloupéen se différencie par des caractères variés de toutes les espèces validées par cet auteur à l'exception de $C$. complanatum $(=C$. marginatum d'après Ukoli). Pour cet auteur, l'un des caractères déterminants est la localisation du complexe génital ; chez $C$. complanatum, le testicule antérieur se trouve dans le tiers moyen du corps et le testicule postérieur dans le tiers postérieur. Il convient de souligner que si cette disposition s'observe bien pour les grands spécimens de Guadeloupe, il n'en est pas de même pour les plus petits chez lesquels le complexe génital peut être situé dans sa quasi-totalité dans la partie antérieure du tiers postérieur. Les premières descriptions de cette espèce, réalisées d'après des spécimens du Nouveau Monde, appellent une remarque pour ce qui concerne la taille des œufs. D’après Osborn (1912), les œufs des adultes nord-américains mesurent $99 \times 66 \mu \mathrm{m}$. Cort (1913) admet un minimum de $91 \times 51 \mu \mathrm{m}$ pour les spécimens d'Amérique du Nord et un maximum de $140 \times 73 \mu \mathrm{m}$ pour ceux d'Amérique du Sud. Ce caractère varierait donc considérablement au sein de cette espèce. La forme guadeloupéenne occupe une position moyenne par la taille de ses œufs $(116 \times 67 \mu \mathrm{m})$.

Mais nous pensons qu'il est nécessaire de faire appel aux caractères larvaires lorsque la position taxonomique de formes très voisines est difficile à établir. Parmi ces caractères, la chétotaxie des cercaires occupe une place de choix; cependant, celle-ci n'a pas fait l'objet d'études détaillées chez les Clinostomidae et il n'est donc pas possible d'effectuer des comparaisons. D'après Krull (1934), la cercaire de C. marginatum possède une soie par furca et six paires de soies latérales sur le tronc caudal ; cette disposition est identique chez la cercaire de $C$. heluans (Ruiz, 1953) ; la queue ne porte pas de soies et la région céphalique porte huit papilles chez C. giganticum (Agarwal, 1960). C. chabaudi (Vercammen Grandjean, 1960) porte huit soies sur un côté du tronc caudal 
et neuf sur l'autre; la région céphalique porte une «couronne apicale de 10 papilles épineuses », l'orifice oral est «bordé par trois papilles orales disposées en triangle », le corps porte «deux papilles pilifères » situées latéralement à la hauteur de la vessie. Euclinostomum heterostomum (Donges, 1974) présente neuf soies médio-dorsales et quatre médio-ventrales sur le tronc caudal ; chaque furca porte huit à dix petites papilles à soie courte et une à trois longues soies.

A l'exception des deux types de soies furcales observées par Donges et non retrouvées chez Clinostomum golvani, l'ensemble de oes données n'est pas en opposition avec ce que nous avons observé, mais s'avère insuffisant pour établir des comparaisons entre espèces ou genres proches.

Nous nous bornerons donc à dégager les caractéristiques chétotaxiques de cette cercaire (comparée à celle des autres Anepitheliocystidia décrits) sans pouvoir préciser si elles sont aussi ou non, celles de toute la famille des Clinostomidae : papilles sensorielles de grande taille; quatre cycles céphaliques; papilles corporelles ventrales et dorsales très peu nombreuses ; les papilles corporelles étant essentiellement représentées par les latérales; papilles du tronc caudal disposées selon six axes: un médio-ventral, deux ventro-latéraux, un médio-dorsal, deux dorso-latéraux; nous rappelons que les papilles caudales médianes sont assez rares chez les Anepitheliocystidia et que seules en possèdent, outre $C$. golvani, les cercaires de Strigeoidea.

Par la grande taille de ses rédies, le parasite guadeloupéen se distingue de Clinostomum complanatum et de ses différents synonymes (tableau I).

Tableau I. Dimensions maximum du corps et du pharynx des rédies chez différentes espèces du genre Clinostomum

\begin{tabular}{|c|c|c|c|c|}
\hline Auteur & Espèce & $\begin{array}{c}\text { Longueur } \\
\text { du corps } \\
(\mu, \mathrm{m})\end{array}$ & $\begin{array}{l}\text { Longueur } \\
\text { du pharynx } \\
(\mu \mathrm{m})\end{array}$ & Mollusque \\
\hline Krull, 1934 & C. marginatum & 490 & 36 & $\begin{array}{l}\text { Helisoma } \\
\text { antrosum }\end{array}$ \\
\hline $\begin{array}{c}\text { Hunter et Hunter, } \\
1934\end{array}$ & C. marginatum & 1140 & - & H. antrosum \\
\hline Cort et al., 1950 & C. marginatum & 1200 & - & H. trivolvis \\
\hline Singh, 1959 & C. piscidium & 492 & $\varnothing 30-33$ & Lymnaea luteola \\
\hline Agarwal, 1960 & C. giganticum & 1150 & 68 & L. acuminata \\
\hline $\begin{array}{c}\text { Nassi et Bayssade- } \\
\text { Dufour, } 1980\end{array}$ & C. golvani & 3100 & 67 & $\begin{array}{l}\text { Biomphalaria } \\
\text { glabrata }\end{array}$ \\
\hline
\end{tabular}

Des différences apparaissent également au niveau des cercaires. Dans le Nouveau Monde, outre la cercaire de $C$. complanatum, on connaît celle de $C$. heluans Braun, 1899 (3). Le cycle de C. heluans dont l'adulte diffère nettement du parasite guadelou-

(3) Il est possible que la cercaire émise à Porto-Rico par Planorbis guadeloupensis (= Biomphalaria glabrata) soit identique à celle de Guadeloupe; mais Marin (1928) qui l'a signalée sous le nom de Cercaria II en a donné une description trop succincte pour pouvoir effectuer des comparaisons. 
péen a été élucidé en Amérique du Sud ; le permier hôte intermédliaire est un Mollusque du genre Australorbis (= Biomphalaria) (Lutz, 1919, 1933, 1934, 1935 ; Ruiz, 1953). Le cycle de $C$. complanatum dont notre adulte est par contre très proche a été étudié en Amérique du Nord par Cort (1913), Krull (1934), Hunter et Hunter (1934, $1935 a$, $1935 b$ ) et Hunter et Hunter (1935); ces derniers auteurs ont décrit un pharynx et 3 à 4 paires de glandes de pénétration chez les cercaines émises par Helisoma antrosum et $H$. campanulatum. D'après Krull (1934), les glandes de pénétration sont au nombre de 4 paires et il n'y a pas d'épines sur le corps en dehors de la région antérieure; les dimensions rapportées par cet auteur, prises également sur des cercaires fixées à chaud dans du formol à $5 \%$, font apparaître que la cercaire nord-américaine possède une queue nettement plus courte (longueur du tronc caudal : 250 à $285 \mu \mathrm{m}$; longueur des fourchons: 75 à $98 \mu \mathrm{m})$. Les différences au niveau des cercaires viennent s'ajouter à celles déjà signalées pour les rédies. C. giganticum Agarwal, 1960 et $C$. piscidium Southwell et Prashad, 1918 sont deux espèces décrites en Inde. Pour Agarwal (1960), C. piscidium est synonyme de $C$. complanatum; Ukoli (1966) considère que cette synonymie doit également s'étendre à $C$. giganticum. La cercaire de $C$. giganticum est émise par un Mollusque Lymnéidé : Lymnaea acuminata ; elle est pharyngée et possède seulement 4 paires de glandes de pénétration (Agarwal, 1960). D'après Singh (1959), le premier hôte intermédiaire de $C$. piscidium est Lymnaea luteola; la cercaire est ici dépourvue de pharynx et possède également 4 paires de glandes de pénétration.

Le parasite guadeloupéen se distingue également de l'espèce décrite en Afrique par Vercammen-Grandjean (1960) sous le nom de Clinostomum chabaudi et dont seuls les stades larvaires sont connus. La cercaire est ici encore émise par un Lymnéidé, Radix natalensis undussumae; si, comme la cercaire guadeloupéenne, elle possède 5 paires de glandes de pénétration, elle est par contre dépourvue de pharynx et ni l'ébauche acétabulaire, ni l'ébauche génitale ne sont mentionnées par l'auteur. Les rédies sont d'assez grande taille (750 à $2100 \mu \mathrm{m}$ de long), mais le pore de naissance est situé à $200 \mu \mathrm{m}$ de l'extrémité antérieure. Contrairement aux espèces déjà citées qui ont un Poisson comme deuxième hôte intermédiaire, celui-ci est ici un Batracien.

L'étude de la spécificité vis-à-vis du Mollusque pourrait constituer d'après nous un moyen de préciser les positions taxonomiques à l'intérieur du genre Clinostomum. Cette spécificité existe puisqu'elle a été démontrée dans le cas de $C$. piscidium; cette espèce dont l'hôte naturel est L. luteola n'infeste pas Indoplanorbis exustus (Singh, 1959). De plus, il convient de remarquer que pour les espèces que nous avons citées, les premiers hôtes intermédiaires sont des Planorbidés en Amérique et des Lymnéidés en Asie et en Afrique. Enfin, on doit noter que C. complanatum, espèce dont le parasite de Guadeloupe est morphologiquement très proche, n'a jamais été signalé dans le Nouveau Monde chez un Biomphalaria ; et qu'inversement $C$. heluans dont le cycle comporte un Biomphalaria est morphologiquement distinct du Clinostomum de Guadeloupe.

L'ensemble des différences relevées au niveau des stades larvaires avec ceux déjà connus dans le genre Clinostomum nous amène à considérer le parasite guadeloupéen comme une nouvelle espèce qui est en particulier distincte de $C$. complanatum telle que cette espèce a été décrite en Amérique du Nord. Nous proposons pour le parasite guadeloupéen le nom de Clinostomum golvani. 
REMERCIEMENTS. - Cette recherche a reçu un support financier de la D.G.R.S.T. et de I'I.N.S.E.R.M.

La partie terrain a été effectuée à la Station de Zoologie de l'I.N.R.A. dans le cadre de l'Action Concertée Bilharziose (Dir. Pr Y.J. Golvan).

\section{Bibliographie}

Agarwal S.M. : Studies on the morphology, systematics and life history of Clinostomum giganticum n. sp. (Trematoda : Clinostomatidae). Ind. J. Helminthol., 1960, 11, 75-115.

Baer J.G.: Note sur un nouveau Trématode, Clinostomum lophophallum sp. nov. avec quelques considérations générales sur la famille des Clinostomidae. Rev. suisse Zool., 1933, 10, 317-342.

Chatton E., Lwoff A. : Imprégnation par diffusion argentique de l'infraciliature des Ciliés marins et d'eau douce après fixation cytologique et sans dessication. C.R. Soc. Biol., 1930, 104, 834-836.

Combes C., Bayssade-Dufour C., Cassone J. : Sur l'imprégnation et le montage des cercaires pour l'étude chétotaxique. Ann. Parasitol. Hum. Comp., 1976, 51, 199-400.

Cort. W.W. : Notes on the trematodes genus Clinostomum. Trans. Amer. micr. Soc., 1913, 32, 169182.

Cort W.W., Ameel D.J., Van der Woude E. : Germinal material in the rediae of Clinostomu $n$ marginatum (Rudolphi). J. Parasitol., 1950, 36, 157-163.

Dollfus R.P.: Trématodes récoltés au Congo Belge par le Professeur Paul Brien. Ann. Mus. Congo Belge (Zoologie), 1950, 1, 1-136.

Donges J. : The life cycle of Euclinostomum heterostomum (Rudolphi, 1809) (Trematoda : Clinostomatidae). Internation. J. Parasitol., 1974, 4, 79-90.

Hunter G.W., Hunter W.S.: The life cycle of the yellow grub of fish, Clinostomum marginatum. J. Parasitol., 1934, 20, 325.

Hunter G.W., Hunter W.S. : Further studies on fish and bird parasites (in a biological survey of the Mohawk-Hudson watershed). 24 Ann. Rep. N. York State Conservation Dept., Suppl., 1935 a, 267-283.

Hunter G.W., Hunter W.S. : Studies on Clinostomum. IV: Notes on the penetration and growth $\cdot \mathrm{t}$ the cercaria of Clinostomum marginatum. J. Parasitol., 1935 b, 21, 411-412.

Hunter W.S., Hunter G.W. : Studies on Clinostomum. II. The miracidium of Clinostomum marginatum. J. Parasitol., 1935, 21, 186-189.

Krull W.H. : Some observations on the cercaria and redia of a species of Clinostomum, apparently C. marginatum (Rudolphi, 1819) (Trematoda : Clinostomidae). Proc. helminthol. Soc. Washington, 1934, 1, 34-35.

L.utz A. : O Schistosomum mansoni e a schistosomatose segundo observaçoes, feitas no Brazil. Mem. Inst. Oswaldo Cruz, 1919, 11, 121-155.

Lutz A. : Notas sobre Dicranocercarias brazileiras. Mem. Inst. Oswaldo Cruz, 1933, 27, 346-376.

Lutz A.: Outro grupo de Trematodes nascendo de Dicranocercarias e outro caso de especie con coecos abrindo para fora. Mem. Inst. Oswaldo Cruz, 1934, 29, 229-248.

Lutz A. : Evolution du Clinostomum heluans. C.R. Soc. Biol., 1935, 118, 289-290.

Marin R.A. : Studies on schistosomiasis (S. mansoni) in Porto Rico. Porto Rico Rev. pub. Hlth. tron. Med., 1928, 3, 397-402.

Osborn H.L. : On the structure of Clinostomum marginatum a trematode parasite of the frog, bass and heron. J. Morphol., 1912, 23, 189-229.

Richard J. : La chétotaxie des cercaires. Valeur systématique et phylétique. Mem. Mus. Nat. Hist. Nat., ser. A, Zoologie, 1971, 67, 1-149.

Ruiz J.M. : Contribuçao ao estudo das formas larvarias de trematoides brasileiros. 5. Descriçao de tres furcocercarias que ocorrem em planorbideos hospedeiros do Schistosoma mansoni. Mem. Inst. Butantan, 1953, 25, 77-89. 
Singh R.N. : Studies on the morphology and life history of Clinostomum piscidium Southwell et Prashad 1918 (Trematoda: Clinostomidae). Proc. Nat. Acad. Sc. India, ser. B, 1959, 29, 12-33.

Ukoli F.M.A. : On Clinostomum tilapiae n. sp. and C. phalacrocoracis Dubois 1931 from Ghana and a discussion on the systematics of the genus Clinostomum Leidy 1856. J. Helminthol., 1966, 40, 187-214.

Vercammen-Grandjean P.H.: Les trématodes du Lac Kivu Sud (Vermes). Ann. Mus. R. Afrique Centrale, Tervuren, n.s., Sc. Zool., 1960, 5, 1-171.

Yamaguti S. : Studies on the helminth fauna of Japan. Part. I. Trematodes of birds, reptiles and mammals. Jap. J. Zool., 1933, 5, 1-134. 